Acta Crystallographica Section A

Foundations of Crystallography

ISSN 0108-7673

Received 6 January 2006

Accepted 30 May 2006

(C) 2006 International Union of Crystallography Printed in Great Britain - all rights reserved

\section{The formation of low-dimensional metal trihalide crystals in carbon nanotubes}

\author{
Mark Wilson $^{\mathrm{a} *}$ and Steffi Friedrichs ${ }^{\mathrm{b}} \neq$ \\ ${ }^{a}$ Department of Chemistry, University College London, 20 Gordon Street, London WC1H OAJ, \\ England, and ${ }^{\mathbf{b}}$ Nanoscience Centre, University of Cambridge, $11 \mathrm{~J}$. J. Thomson Avenue, Cambridge \\ CB3 OFF, England. Correspondence e-mail: m.wilson@ucl.ac.uk
}

\begin{abstract}
Molecular dynamics computer simulation models are employed to study the direct filling of single-walled carbon nanotubes (which vary in diameter) with an archetypal metal trihalide, $\mathrm{LaCl}_{3}$. The use of relatively simple potential models allows the investigation of details of both the atomistic filling mechanism and the thermodynamic factors controlling the formation. The resulting low-dimensional crystallites are analysed with respect to bulk crystal structures and compared to experimental high-resolution transmission-electron-microscopy images by simulation of equivalent micrographs from one of the obtained potential models, resulting in excellent agreement between the simulated and experimental images.
\end{abstract}

\section{Introduction}

Since the discovery of carbon nanotubes (Ajayan \& Ebbesen, 1997; lijima, 1991; Bethune et al., 1993), numerous potential applications have been suggested. One strategy is to use the carbon nanotubes themselves, controlling useful properties via their radii and morphologies. An alternative strategy is to use the carbon tubes as effective templates to grow new materials (of reduced dimensionality) which may be effectively impossible to grow in the absence of such templates. The advantage of the latter scheme lies in the potential flexibility in controlling the chemical composition of the non-carbon-based low-dimensional crystals. Significant experimental progress has been made in filling carbon nanotubes over the past decade with materials such as $\mathrm{NiO}, \mathrm{PbO}, \mathrm{Bi}_{2} \mathrm{O}_{3}, \mathrm{~V}_{2} \mathrm{O}_{5}$ and $\mathrm{MoO}_{3}$ (Ajayan, Ebbesen et al., 1993; Ajayan, Ichihashi \& Iijima, 1993; Tsang et al., 1994; Ajayan et al., 1995; Chen et al., 1996), $\mathrm{UCl}_{4}$ (Sloan et al., 1998), $\mathrm{AgCl} / \mathrm{AgBr}$ (Sloan et al., 1999; Sloan, Terrones et al. 2002), KI (Sloan et al., 2000; Meyer et al., 2000), $\mathrm{BaI}_{2}$ (Sloan, Grosvenor et al., 2002), $\mathrm{CoI}_{2}$ (Philp et al., 2003) and $\mathrm{Sb}_{2} \mathrm{O}_{3}$ (Friedrichs et al., 2001). Metal salts represent a useful class of filling material as their surface tension tends to be low enough so as not to crush the inserted tube. High-resolution transmission-electron-microscopy (HRTEM) studies show the structures formed inside the carbon nanotubes to be crystalline despite the tubes filling directly from the molten salts. However, whilst a wide range of materials has been inserted experimentally, a full theoretical understanding of the thermodynamics and kinetics of such crystallite formation is lacking. In addition, material structures grown to date and successfully identified are directly related to bulk crystal structures (via, for example, relatively simple distor-

$\ddagger$ Present address: TTP (The Technology Partnership plc), Melbourn Science Park, Cambridge Road, Melbourn SG8 6EE, England. tions). However, it is not clear that such low-dimensional crystallites (which may be thermodynamically or kinetically stable) should retain such a simple relationship with bulk crystals. Furthermore, the factors controlling the formation and growth of such crystallites must be understood if true control over their physical properties is to be attained. Simulation models offer a potentially unique insight into the formation of these crystals. Work to date has focused on the $M X$ stoichiometry (Wilson \& Madden, 2001; Wilson, 2002a,b, 2004). Simple potential models, in which the carbon nanotube is treated atomistically but is fixed in space, are able to capture the experimentally observed structures (Wilson \& Madden, 2001; Wilson, 2002a). Furthermore, they predict entirely new classes of inorganic nanotubes (INT) whose relationship with the bulk crystal structures is less easy to define (Wilson, 2002b, 2004).

In this paper, we shall focus on a system of $M X_{3}$ stoichiometry $\left(\mathrm{LaCl}_{3}\right)$ in order to make contact with recent HRTEM experiments (Friedrichs et al., 2004, 2005; Friedrichs \& Green, 2005). The aim is to promote a symbiotic relationship between simulation and experiment, in which such modelling not only helps to explain existing experimental results but also helps to guide future experimental direction. Simulating the filling of the nanotubes by a system with this stoichiometry presents a number of challenges. The crystal structure identified from the HRTEM studies indicates the possible formation of highly asymmetric structures within the nanotube with resulting relatively large in-tube free volumes, unlike in the $M X$ stoichiometry in which the preferred crystal structures adopt a highly space filling motif (Wilson, 2002b, 2004). Furthermore, there are a number of bulk crystal structures which may be energetically accessible and which, therefore, may provide templates for in-tube crystal formation. Finally, recent experimental observation suggests an in situ reduction of the 
$M X_{3}$ system to $M X_{2}$ (with an associated formation of pure $X_{2}$ ). As a result, it is of interest to test whether a simulation model based exclusively on the $M($ III) valence state can reproduce the experimental observations.

Filling experiments have been mainly concerned with triiodide systems, such as $\mathrm{LaI}_{3}$, owing to their high contrast in HRTEM analysis. The present modelling project will focus on the equivalent chloride, $\mathrm{LaCl}_{3}$, which exhibits a crystal structure map analogous to that of the iodides (Pettifor, 1994). Furthermore, the potential models for the chlorides have been developed over a significant period of time and have been shown to account for system properties over a wide range of phase space and to be highly transferable to systems with different cations (Hutchinson et al., 1999, 2001; Glover \& Madden, 2004; Okamoto \& Madden, 2005). As a result, we are confident that these potential models will be capable of reproducing the characteristic crystal structures that may be found in the carbon nanotubes.

\section{Potential models}

The fundamental forms used in the simulation models have been described in detail elsewhere (Madden \& Wilson, 1996, 2000; Hutchinson et al., 1999, 2001; Glover \& Madden, 2004; Okamoto \& Madden, 2005). The $M \mathrm{Cl}_{3}$ salts are modelled as ensembles of formal (valence) charges in which the ions interact through a Born-Mayer potential:

$$
U_{i j}\left(r_{i j}\right)=B_{i j} \exp \left(-a_{i j} r_{i j}\right)+\frac{Q_{i} Q_{j}}{r_{i j}}-\frac{C_{6}^{i j}}{r_{i j}^{6}},
$$

where $Q_{i(j)}$ is the charge on ion $i(j), B_{i j}$ and $a_{i j}$ control the shortrange repulsion, and $C_{6}^{i j}$ is the dipole-dipole dispersion term. The pair potential is augmented with a description of the (many-body) dipolar anion polarization via a polarizable-ion model (PIM) (Madden \& Wilson, 1996, 2000). The PIM requires two further parameters; the anion polarizability, $\alpha$, which controls the ion response to electric fields resulting from charge motion, and the short-range damping parameter, which controls the response of the anion to short-range interactions with the nearest-neighbour cations. The parameters used in the present work are taken from Hutchinson et al. (1999, 2001), Glover \& Madden (2004) and Okamoto \& Madden (2005) and have been derived for a range of metal chlorides, which can be considered as differing only in the cation radius, by reference to both crystal and liquid structures (Hutchinson et al., 1999, 2001; Glover \& Madden, 2004; Okamoto \& Madden, 2005), the latter as determined from neutron diffraction studies (Wasse \& Salmon, 1999). The ions interact with the (fixed atomistic) carbon nanotubes through simple Lennard-Jones potentials. The potential parameters are derived by reference to the isoelectronic noble gases as described previously (Wilson, 2002a).

Molecular dynamics simulations are performed at constant temperature and pressure in order to directly simulate the filling process. A temperature of $1500 \mathrm{~K}$ and zero pressure are chosen, at which state point the ion diffusivities are $\sim 10^{-5} \mathrm{~cm}^{2} \mathrm{~s}^{-1}$. A cylindrical section is removed from an equilibrated liquid configuration (containing 1728 ions) and replaced with a single-walled carbon nanotube (C-SWNT) of the required $(n, m)$ morphology (Saito et al., 1998) generated in the standard fashion by folding graphene sheets along a specified chiral vector, $\mathbf{C}_{h}=n \mathbf{a}_{1}+m \mathbf{a}_{2}$ (Saito et al., 1998), where $\mathbf{a}_{1}$ and $\mathbf{a}_{2}$ are the unit-cell vectors. Both ends of the tube are blocked with graphene sheets in order to allow equilibrium to be re-established without the tube starting to fill. The ends are then removed and tube filling is allowed to start. This procedure is adopted as it allows us to directly sample possible crystal structures from the (confined environment) phase diagram without reference to known bulk structures. Once possible crystallites have been identified, unit cells can be determined which allows idealized crystals to be generated and allows the $(0 \mathrm{~K})$ thermodynamic relationships, as a function of pore radius, to be determined.

\section{Results}

Fig. 1 (inset) shows a typical time evolution for the number of ions entering a single-walled carbon nanotube $[$ the $(18,0)$

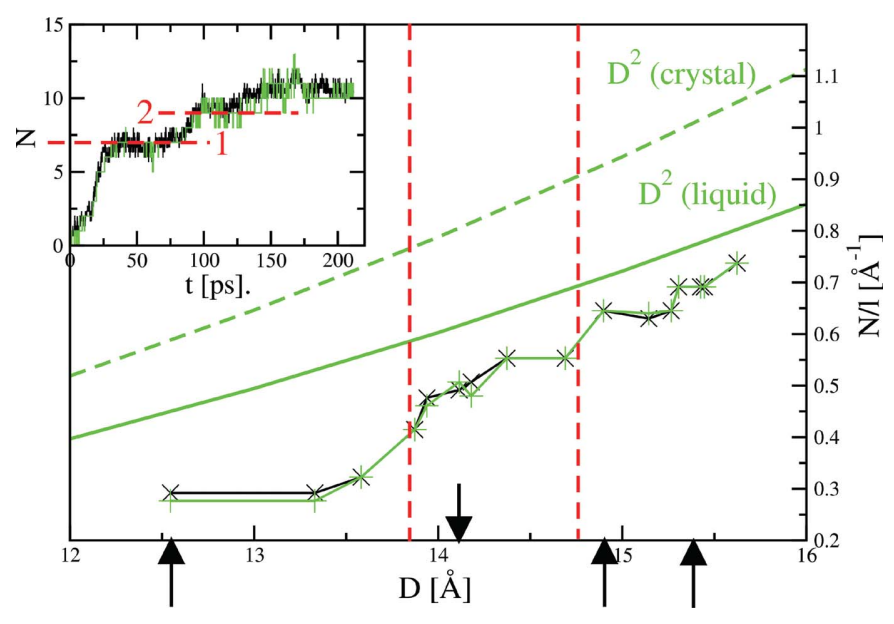

Figure 1

Main panel: The number of cations $(x)$ and anions $(+)$ present in the carbon nanotubes at complete filling, per unit length, as a function of diameter, $D$. The number of anions is divided by three to account for electroneutrality. The solid and dashed curves show the predicted number of ions present from a simple bulk filling analysis, assuming crystalline and liquid densities, respectively. The vertical dashed lines highlight the 'boundaries' between the construction of single-, double- and triplecation-centred polyhedral crystal structures, respectively. The arrows below the $x$ axis indicate the diameters of the $(16,0),(18,0)$ and $(19,0)$ carbon nanotubes which are used as examples of the three filling regimes in the main text. Inset: An example filling profile for the $(18,0)$ carbon nanotube showing the number of ions inside the nanotube, normalized by the number of ions per molecule, as a function of time. The black and green lines are for the cations and anions, respectively. The dashed lines represent two plateaus corresponding to the formation of relatively stable 'intermediate' structures on filling. The points along the $x$ axis in the main panel correspond to the diameters of specific C-SWNTs. From low to high diameter, these are the $(16,0),(17,0),(10,10),(16,3),(14,6),(18,0)$, $(17,2),(16,4),(15,6),(19,0),(17,4),(15,7),(19,1),(18,3),(16,6)$, $(17,5)$, with diameters $12.53,13.31,13.56,13.85,13.92,14.09,14.16,14.35$, $14.67,14.87,15.12,15.24,15.28,15.40,15.42,15.64 \AA$ A, respectively. 
C-SWNT is shown here]. The number of ions rises, on a nanosecond time scale, to a plateau, corresponding to a filling velocity $\sim 7 \mathrm{~m} \mathrm{~s}^{-1}$. The number of anions in the tube, divided by the number of anions per formula unit to retain electroneutrality, mirrors the number of cations, indicating that the material filling the tube retains charge neutrality throughout. In addition to the long-time plateau, the filling shows two further plateaus at $\sim 30$ and $\sim 100 \mathrm{ps}$, respectively. The 'residence times' of these two plateaus $(\sim 50$ and $\sim 30$ ps, respectively, corresponding to $\sim 80000$ and $\sim 50000$ molecular dynamics time steps) indicate the possible existence of well defined, relatively stable, pre-cursor structures.

Fig. 1 (main panel) shows the number of anions and cations in the nanotubes at equilibrium (in the long-time limit). Again, the number of anions, divided by three to take account of electroneutrality, indicates that charge-neutral species are being formed in this range of pore radii. Distinctive 'jumps' are seen in this function at $\sim 13.9$ and $14.8 \AA$, respectively (indicated by the dashed lines in the figure). If the internal structure were simply based on the bulk liquid, then the number of ions inside each nanotube would simply be governed by the liquid density, $\rho_{l}$, and the effective internal nanotube free volume. In this case, the number of ions inside the tube per unit length is $N / l=\frac{1}{4} \rho_{l} \pi D_{e}^{2}$, where $D_{e}$ is the internal tube diameter $\left(\equiv D-\sigma_{\mathrm{CC}}\right.$, where $D$ is the carbon nanotube diameter and $\sigma_{\mathrm{CC}}$ is a $\mathrm{C}$-atom diameter). Fig. 1 shows the number of ions at each nanotube diameter predicted from this analysis. The number of ions filling the tubes from the direct filling simulations are consistently smaller than those predicted simply from the liquid density. Furthermore, as also shown in Fig. 1, the number of ions predicted using the (higher) bulk crystal density (i.e. assuming

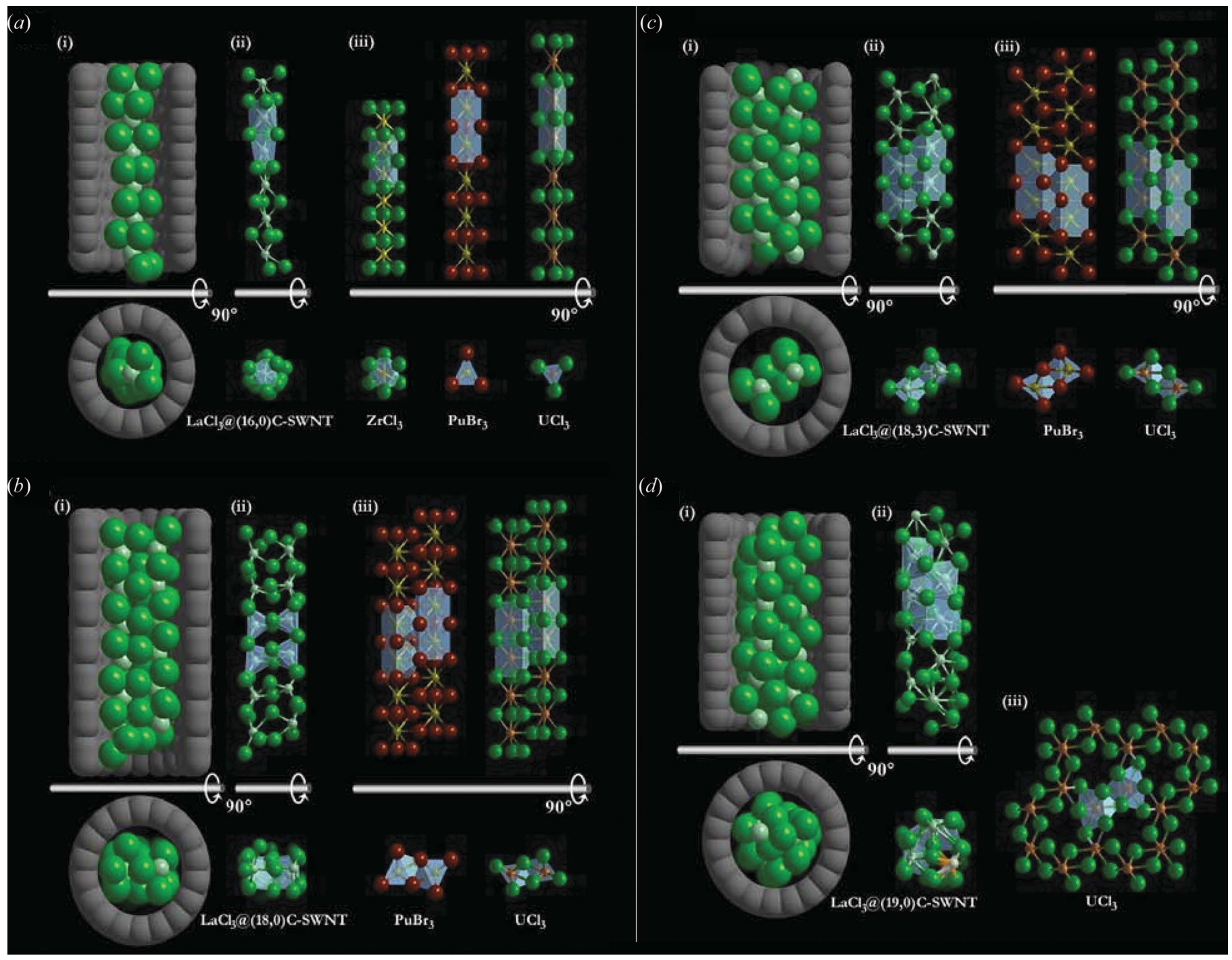

Figure 2

Composite picture illustrating the relation between the bulk structures of $\mathrm{ZrCl}_{3}, \mathrm{PuBr}_{3}$ and $\mathrm{UCl}_{3}$ and the $\mathrm{LaCl}_{3}$ filling material inside (a) a (16, 0 ) C-SWNT, $(b)$ an $(18,0)$ C-SWNT, $(c)$ an $(18,3)$ C-SWNT and $(d)$ a $(19,0)$ C-SWNT, respectively. Each filling composite model is compared to an equivalent fragment of bulk $\mathrm{ZrCl}_{3}$ and/or $\mathrm{PuBr}_{3}$ and/or $\mathrm{UCl}_{3}$; all the crystal fragments are reproduced to relative scale and the main polyhedra are indicated in pale blue. In the upper parts of all parts (i), the top half of the carbon nanotube has been omitted for clarity. 
the internal structure to be crystalline and space filling) is even further removed from the actual quantity of material observed. This difference is consistent with the formed internal structures having a significant degree of 'free volume' - the internal structures formed appear not to be space filling. This behaviour is distinct from that observed for the $M X$ stoichiometry in which the amount of material present in the internal crystallites broadly follows the bulk density (Wilson, 2004). This analysis is, at present, based on the available volume present within the carbon nanotube. It does not consider the interaction between the inserted ions and the $\mathrm{C}$ atoms.

Fig. 2 shows molecular graphics 'snapshots' of the average crystal structure obtained in the $(16,0),(18,0),(18,3)$ and $(19,0)$ carbon nanotubes (diameters: 12.53, 14.09, 15.40 and $14.87 \AA$, respectively), which are typical of those found in the plateaus in Fig. 1 (and indicated in Fig. 1 by the black arrows). The structures are generated by time-averaging the ion positions over the final $5 \mathrm{ps}$ of the molecular dynamics simulations [i.e. within the long-time plateaus in Fig. 1 (inset) and long enough so as to effectively average out the local vibrational modes].

Detailed structural analyses of the crystal fragments formed within the $(16,0),(18,0),(18,3)$ and $(19,0)$ single-walled carbon nanotubes reveal the complexity of relationships between the size and chirality parameters of the surrounding C-SWNTs and the resulting structure adopted by the filling material. In all cases, however, the crystal structure of the $\mathrm{LaCl}_{3}$ fragment could be related to at least one of the bulk structures of $\mathrm{ZrCl}_{3}, \mathrm{UCl}_{3}$ or $\mathrm{PuBr}_{3}$ (Larsen et al., 1982; Wells, 1984; Zachariasen, 1948 ${ }^{\mathbf{1}}$ ); the main similarities and differences between these three structures are illustrated in Fig. 3: bulk $\mathrm{ZrCl}_{3}$ consists of individual chains of face-sharing $\mathrm{ZrCl}_{6}$ octahedra, running along [100], while the equivalent chains in both $\mathrm{PuBr}_{3}$ (parallel to [010]) and $\mathrm{UCl}_{3}$ (parallel to [001]) consist of doubly capped trigonal bipyramids that are linked within the $x z$ plane to form infinite $\mathrm{PuBr}_{3}$ layers and triply capped trigonal bipyramids additionally linked within the $x y$ plane to form a three-dimensional network of $\mathrm{UCl}_{3}$.

Fig. 2(a) shows the structure formed within the $(16,0)$ C-SWNT; it consists of a single-cation chain of face-sharing $\mathrm{LaCl}_{6}$ octahedra, distorted towards trigonal bipyramidal shapes. This dynamically formed chain can be considered to be derived from a number of different bulk crystal structures: firstly, the fragment shows strong similarity with a distorted version of an individual [001] chain of the $\mathrm{ZrCl}_{3}$ crystal structure (Larsen et al., 1982; Wells, 1984); secondly, the fragment resembles a distorted $M X_{3}$ chain, which runs along the [100] direction of the $\mathrm{PuBr}_{3}$ structure (Zachariasen, 1948; Wells, 1984) and, thirdly, a chain running along the [001] direction of bulk $\mathrm{UCl}_{3}$ (Wells, 1984; Schleid et al., 1987).

Fig. 2 shows a composite picture illustrating the relationship between the chain of $\mathrm{LaCl}_{3}$ dynamically formed within the

\footnotetext{
${ }^{\mathbf{1}}$ The originally reported space group $\mathrm{Ccmm}$ was transformed into $\mathrm{Cmcm}$ for the discussion in this paper.
}

$(16,0)$ C-SWNT [Figs. 2(a)(i) and (ii)] and comparable crystal fragments of the bulk structures of $\mathrm{ZrCl}_{3}, \mathrm{UCl}_{3}$ and $\mathrm{PuBr}_{3}$

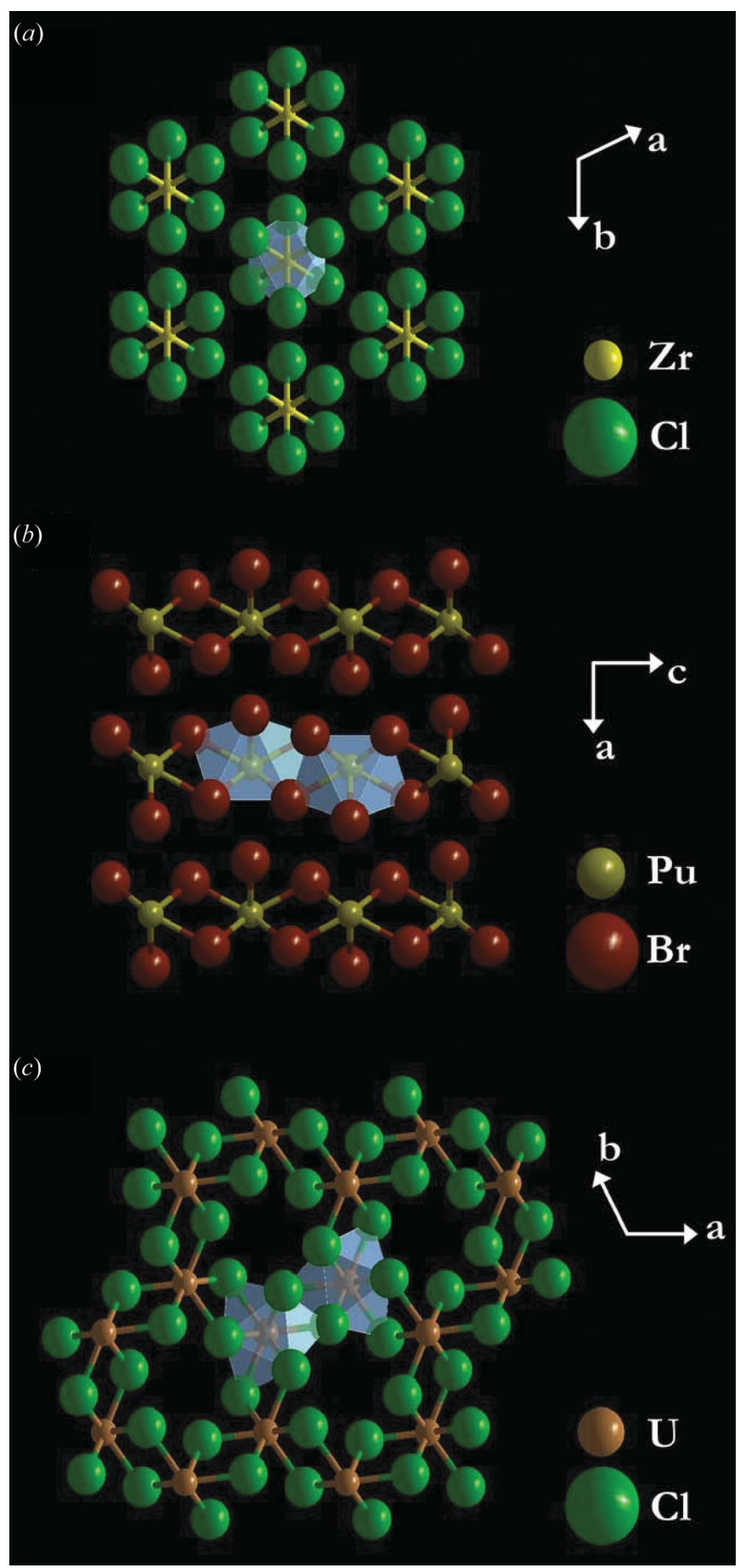

Figure 3

Composite picture illustrating the similarities and differences between the bulk structures of $(a) \mathrm{ZrCl}_{3}$, (b) $\mathrm{PuBr}_{3}$ and (c) $\mathrm{UCl}_{3}$ (Zachariasen, 1948; Larsen et al., 1982; Wells, 1984; Schleid et al., 1987). Bulk $\mathrm{ZrCl}_{3}$ consists of individual $\left(M X_{6}\right)_{n}$ chains, while $\mathrm{PuBr}_{3}$ is a layered structure [i.e. the $\left(M X_{6}\right)_{n}$ chains are connected within the $x z$ plane] and $\mathrm{UCl}_{3}$ consists of a three-dimensional network [i.e. the $\left(M X_{6}\right)_{n}$ chains are connected within both the $x z$ plane and the $x y$ plane] (with $M=\mathrm{Zr}, \mathrm{Pu}, \mathrm{Br}$ and $X=\mathrm{Cl})$. In each case, the $\left(M X_{6}\right)_{n}$ chains and their respective polyhedral geometries are highlighted via the addition of polyhedron faces. 
[Fig. 2(a)(iii)]; these consist of face-sharing octahedra in the case of $\mathrm{ZrCl}_{3}$, while equivalent fragments of $\mathrm{PuBr}_{3}$ and $\mathrm{UCl}_{3}$ are composed of face-sharing trigonal bipyramids. Fig. 2(a)(i) displays the dynamically modelled $\mathrm{LaCl}_{3} @(16,0)$ C-SWNT composite in both a side-on (upper part) and an end-on (i.e. viewed along the tube axis; lower part) projection; in this model, the atoms are drawn to scale (i.e. all ions and atoms are represented by spheres of sizes corresponding to the oxidation states in an uncharged $\mathrm{LaCl}_{3}$ crystal fragment and C-SWNT, respectively) (http://www.webelements.co.uk). The end-on view highlights the significant free volume present around the crystallite, consistent with the conclusion drawn for the analysis of Fig. 1 (see above). The mean metal-metal bond length of the dynamically grown crystallite is $3.99 \AA$ with an $M-X-M$ bond angle of $92^{\circ}$. This separation is comparable with those typical of the $\mathrm{UCl}_{3}$ and $\mathrm{PuBr}_{3}$ structures, but considerably longer than those observed in $\mathrm{ZrCl}_{3}$ (in $\mathrm{ZrCl}_{3}$ itself, for example, the $\mathrm{Zr}-\mathrm{Zr}$ separation is $3.07 \AA$ with an average $\mathrm{Cl}-\mathrm{Zr}-\mathrm{Cl}$ bond angle of $90^{\circ}$, where significant metal-metal bonding is present.

In the $(18,0) \mathrm{C}$-SWNT, the filling fragment exhibits two cations in the tube cross section [see Fig. 2(b)(i)]; similar to the $\mathrm{LaCl}_{3}$ filling inside the $(16,0)$ C-SWNT, Fig. 2(b)(i) highlights the significant fraction of free volume present inside the $(18,0)$ nanotube. The structure of the filling material can be consid- ered as two linked chains of edge-sharing trigonal bipyramids (six-coordinate cations); in contrast, equivalent doublechained fragments extracted from the crystal structures of $\mathrm{PuBr}_{3}$ and the $\mathrm{UCl}_{3}$ [Fig. 2(b)(iii)] show both edge- and facesharing singly capped bipyramids (i.e. seven-coordinate cations; face-sharing within the chains, edge-sharing between the chains) (Zachariasen, 1948; Schleid et al., 1987). In both the bulk structures, the extended coordination sphere and face-sharing arrangement of polyhedrons are enabled by the regular staggered arrangement of the cations of both chains with respect to each other; this arrangement enables $1 / 3$ of the halide ions to adopt a trivalent state, bridging between both sides of the chain, while the parallel alignment of cations (hereafter referred to as the 'ladder' arrangement) spatially inhibits higher-valency states of the anions, so that all $\mathrm{Cl}$ atoms are divalent. The $\mathrm{LaCl}_{3}$ fragment dynamically formed inside the $(18,0)$ C-SWNT exhibits a structure that can be described as a hybrid between the two arrangements: the cations are slightly staggered with regard to each other, but the staggering angle is rather small $(\mathrm{ca} \mathrm{20})$ compared to that in the bulk structures of $\mathrm{PuBr}_{3}$ and $\mathrm{UCl}_{3}\left(\right.$ i.e. $45^{\circ}$ ), so that the bridging $\mathrm{Cl}$ ions are divalent $(c f . \S 3.1)$.

Inside the $(18,3) \mathrm{C}$-SWNT, however, the cations within the dynamically formed double-chained $\mathrm{LaCl}_{3}$ fragment, shown in Fig. 2(c)(i), are arranged in a regular staggered alignment,
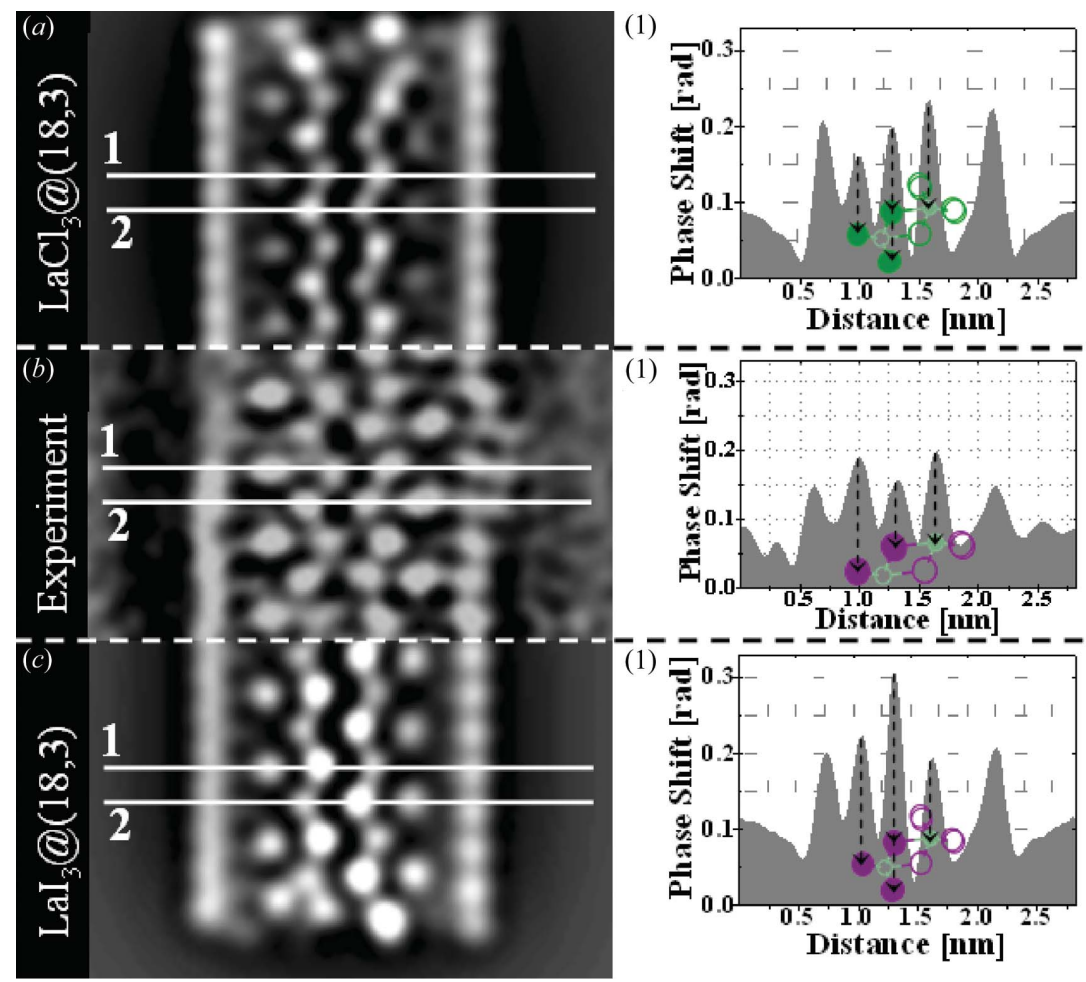

(2)
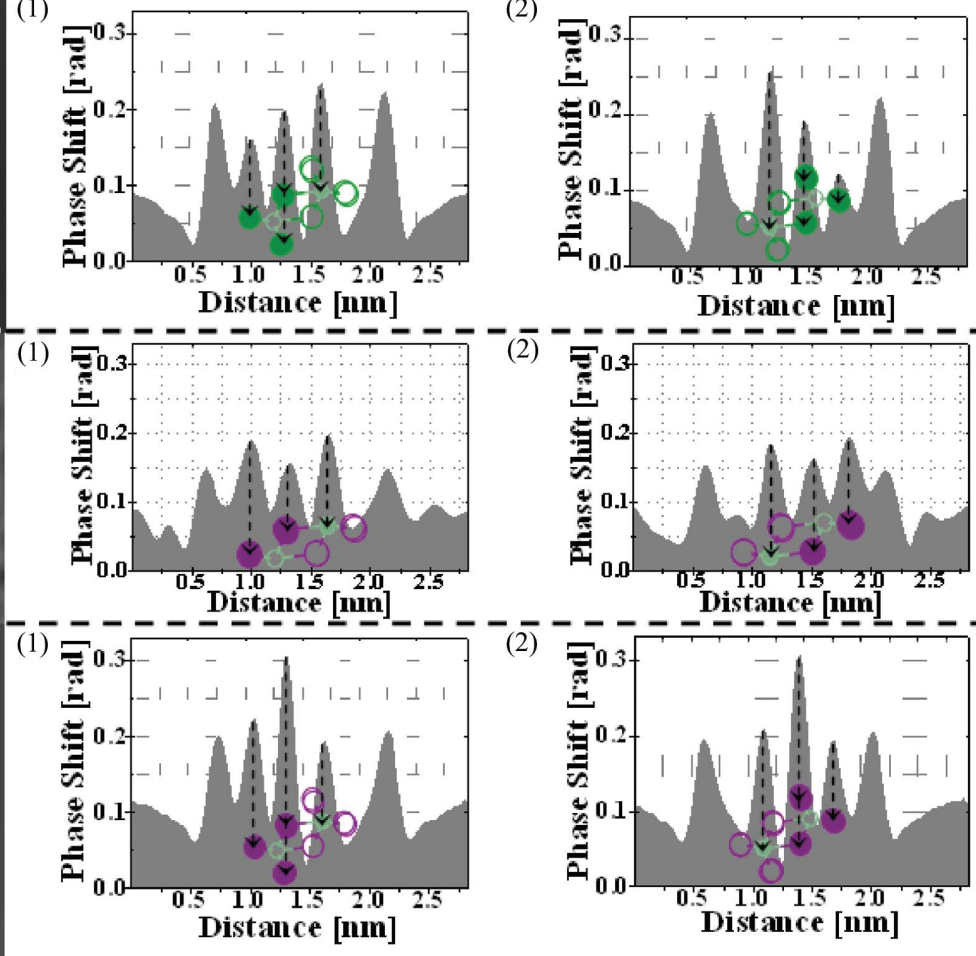

Figure 4

Simulated multislice HRTEM phases of $(a)$ the dynamically modelled $\mathrm{LaCl}_{3} @(18,3)$ C-SWNT system, $(b)$ an experimentally observed LaI $@$ @(18, 3$)$ C-SWNT encapsulation composite (Friedrichs et al., 2005), and (c) the model of an LaI @ $(18,3)$ C-SWNT system, constructed from the model used in $(a)$ by substituting all $\mathrm{Cl}$ atoms with I atoms, in order to simulate a phase contrast matching that of $(b)$. The white lines (1) and (2) indicate two single-pixel line profiles displayed to the right of the respective image. The stylized crystal fragment and black arrows superimposed onto the profiles illustrate the number and nature of the atom columns that give rise to the respective phase shifts (i.e. full circles represent the atoms stacked underneath each peak, empty circles represent connecting atoms lying within an adjacent three-membered row). 
identical to that exhibited by the equivalent fragment of bulk $\mathrm{PuBr}_{3}$ and $\mathrm{UCl}_{3}$ [see Fig. 2(b)(iii)]. Similarly, the cations in the $\mathrm{LaCl}_{3} @(18,3)$ C-SWNT are coordinated by seven anions in the form of a singly capped bipyramid, as illustrated in Fig. 2(c)(ii); these polyhedra are face-sharing (edge-sharing) parallel (perpendicular) to the SWNT axis. The structure adopted within the $(18,3) \mathrm{C}$-SWNT is thus entirely commensurate with the structure of equivalent fragments of both bulk $\mathrm{PuBr}_{3}$ and bulk $\mathrm{UCl}_{3}$. Furthermore, the metal-metal spacing observed within the dynamically formed $\mathrm{LaCl}_{3}$ fragment (average value $4.10 \pm 0.09 \AA$ ) is commensurate with that found in bulk $\mathrm{PuBr}_{3}$ [i.e. 4.11 (3) $\AA$ ] [cf. metal-metal distance in $\mathrm{UCl}_{3}: 4.3243$ (3) $\mathrm{A}$. The $\mathrm{LaCl}_{3} @(18,3) \mathrm{C}$-SWNT model was thus used to simulate HRTEM images, which can be directly compared to the previously experimentally observed LaI $_{3} @$ SWNT systems (Friedrichs et al., 2004, 2005; Friedrichs $\&$ Green, 2005). A detailed discussion can be found in $\S 3.1$.

In the $(19,0) \mathrm{C}$-SWNT, the $\mathrm{LaCl}_{3}$ structure formed has three cations per tube cross section, as illustrated in Fig. $2(d)(i)$. Unlike the crystal fragments formed within the three smaller nanotubes, the fragment found to form in the $(19,0)$ C-SWNT is not directly equivalent to small sections of bulk
$M X_{3}$ crystal structures (with $M=\mathrm{Zr}, \mathrm{Pu}, \mathrm{U}$ and $X=\mathrm{Cl}, \mathrm{Br}$ ); its main structural motifs can, however, be related to distorted components of the $\mathrm{UCl}_{3}$ crystal structure. This relation is illustrated in Figs. 2(d)(ii) and (iii): Fig. 2(d)(ii) displays the complex filling fragment in side-on and end-on views, respectively, illustrating four polyhedra (partially incomplete) similar to those found in the bulk structure of $\mathrm{UCl}_{3}$. Within the linked polyhedra, the cations of the $\mathrm{LaCl}_{3}$ fragment adopt the staggered arrangement that was found to be necessary to yield complex linked capped bipyramids within the bulk structures of $\mathrm{PuBr}_{3}$ and $\mathrm{UCl}_{3}\left[c f\right.$. the $\mathrm{LaCl}_{3}$ fragments formed within the $(18,0)$ and the $(18,3)$ C-SWNT]. Two of the three cation chains thus form a distorted version of the $M_{2} X_{6}$ double chain found in the $\mathrm{UCl}_{3}$ structure to run parallel to the [001] direction. These double chains are interconnected to form a three-dimensional network via outward-pointing $\mathrm{Cl}-\mathrm{U}$ bonds [highlighted in Fig. 2(d)(iii) as dashed white bonds]. In the dynamically formed fragment, the third cation chain is added to yield a regular triangular arrangement of cations in the cross section, moving inwards those bonds that connect each double chain in bulk $\mathrm{UCl}_{3}$ to a three-dimensional network; they are highlighted as dashed white bonds in the end-on view in Fig. 2(d)(ii). This bond folding locally increases the coordination number of the central $\mathrm{Cl}$ ions to an unusual quarto-valency; the additional bond (both inwards towards the central $\mathrm{Cl}$ ions and outwards towards additional $\mathrm{Cl}$ anions) are highlighted as dashed orange in the end-on view of Fig. 2(d)(ii).

Note that, in all four cases, the crystallites formed are charge neutral, that is, the $M X_{3}$ stoichiometry is maintained.

\subsection{Comparison to HRTEM experi- ments}

The dynamically formed model of $\mathrm{LaCl}_{3} @(18,3)$ C-SWNT, shown in Fig. 4(a), can be related to a restored HRTEM phase (image) of an $\mathrm{LaI}_{x} @(18,3)$ C-SWNT encapsulation composite, shown in Fig. 4(b) (Friedrichs et al., 2004, 2005; Friedrichs \& Green, 2005). [A full description of the experimental and physical methods applied for the preparation, focal series acquisition and characterization of the $\operatorname{LaI}_{x} @(18,3)$ C-SWNT composite can be found in Friedrichs et al. (2005).] In order to obtain a comparable simulated HRTEM phase (image) of the $\mathrm{LaCl}_{3} @(18,3)$ C-SWNT model, the calculated model was rotated into 
the proposed viewing direction [i.e. the side-on view displayed in the upper part of Fig. 2(c)(i)], and its projected ion positions were subjected to a standard multislice algorithm (Cowley \& Moodie, 1957; Goodman \& Moodie, 1974), applying code developed by Kirkland (1998). The obtained raw HRTEM simulations were furthermore subjected to the contrast transfer function obtained during the restoration of the experimental focal series, in order to account for the experimental limitations of the imaging and restoration process. Fig. 4(a) shows the thus obtained simulated phase of the dynamically formed $\mathrm{LaCl}_{3} @(18,3)$ C-SWNT composite. For the sake of clear comparability with the experimental HRTEM phase, the $\mathrm{Cl}$ ions in the dynamically formed $\mathrm{LaCl}_{3} @(18,3)$ C-SWNT model were exchanged for I atoms and the resulting $\mathrm{LaI}_{3} @(18,3)$ C-SWNT phase simulation is displayed in Fig. 4(c). Direct comparison between the simulated and experimental HRTEM images emphasizes the main structural similarities and intensity differences between the three systems: the viewing direction of the filling fragment is identical in all three cases, corresponding to a projection along [302] of the bulk structure of $\mathrm{PuBr}_{3}$. In projection, each filling fragment consists of a set of three-membered rows, alternately aligned along the left (1) or right (2) C-SWNT wall; the white lines running across these three-membered rows mark the traces of single-pixel line profiles, plotted to the right of the respective image. In the restored experimental phase and multislice simulation, the intensity of the phase shift (grey value) produced by each projected atom column is proportional to the electron density in projection and it is possible, therefore, to extract information about the number and type of atoms in each row; the interpretation of this complex contrast variation is illustrated in the line profiles in Fig. 4 by the superimposed black dashed arrows and respective crystal fragments (i.e. full circles represent the atoms stacked underneath each peak, empty circles represent connecting atoms lying within adjacent three-membered rows). ${ }^{2}$ In the case of the dynamically modelled $\mathrm{LaCl}_{3} @(18,3)$ C-SWNT, the highest peaks in both line profiles can be assigned to the La atom, while the $\mathrm{LaI}_{3} @(18,3) \mathrm{C}$-SWNT system displays a much higher peak for the contrast created by two superposed I atoms. The experimentally imaged $\mathrm{LaI}_{x} @(18,3)$ C-SWNT system, however, displays an entirely different contrast altogether; detailed analysis of the restored phase, together with additional microscopy and spectroscopy (such as scanning transmission electron microscopy, STEM, and electron-energy-loss spectroscopy) revealed that the filling fragment has an $\mathrm{LaI}_{2}$ stoichiometry but adopts the structure of bulk $\mathrm{LaI}_{3}$ (Friedrichs et al., 2005). The line profiles of the experimental image thus exhibit the lowest peak in the centre of each three-membered row, caused by the projection of only one I atom [cf. two superposed I atoms in Fig. 4(c)].

\footnotetext{
${ }^{2}$ Note that the absolute intensities of the simulated phase shifts are significantly higher than those of the experimental phase shifts. This discrepancy between the contrast of simulated and experimental conventional high-resolution TEM (HRTEM) images has been observed before but remains relatively poorly understood (Boothroyd, 1998).
}

The direct comparison of phases and line profiles reveals an excellent match between the structures of the dynamically modelled La $X_{3} @(18,3)$ C-SWNT and the restored experimental HRTEM phase of the previously characterized $\mathrm{LaI}_{2} @(18,3)$ C-SWNT encapsulation composite (Friedrichs et al., 2004, 2005; Friedrichs \& Green, 2005).

\subsection{Mechanisms of formation}

The simulation of the direct filling of the nanotubes allows us to understand the mechanisms by which the crystallites form (information that is difficult to obtain from experiment). Fig. 5(a) shows a series of molecular graphics 'snapshots' for the filling of the $(16,0)$ carbon nanotube. After $\sim 18$ ps of molecular dynamics, a small crystallite has formed in the C-SWNT opening. At $\sim 62 \mathrm{ps}$, the crystallite has pushed into the nanotube by a further unit-cell length. The advance of the cation chain into the nanotube is facilitated by the motion of the accompanying anions. At $\sim 18 \mathrm{ps}$, the cation furthest into the nanotube is coordinated to only four nearest-neighbour anions and, as a result, can be considered as under-coordinated. The under-coordination of this cation provides a driving force (to attain a 'full' first coordination shell) to pull further material into the tube. The required increase in cation coordination number is achieved by rotations about the cation at the pore opening. The rotation acts to drag further material to the tube opening and hence pushes the growing crystallite further into the nanotube. The highlighted anions in Fig. 5(a) indicate that the face-centred unit, once formed within the nanotube, retains its integrity as a further crystallite is formed. As a result, it is the rotations at the mouth of the carbon nanotube that act to introduce new material to the tube. Once these ions are arranged in the face-sharing chains, they are pushed further into the tube by further rotations at the tube opening. However, the anions in each individual face-sharing unit do interchange in the tube, that is, rotations about the cation-centred chain appear relatively facile. Note that the rotational motions are facilitated by the carbon nanotube effectively disrupting the liquid three-dimensional network structure. In the bulk liquid, such rotations require significant correlated ion motion owing to the high network connectivity. The presence of these motions, not present in the bulk material, may have implications for the vibrational spectra of the in-tube material.

Fig. 5(b) shows a series of snapshots for the filling of the $(18,0)$ C-SWNT. The initial structure to grow (at $\sim 54 \mathrm{ps}$ ) is the single-cation chain of face-sharing octahedra [as seen in the filling of the $(16,0) \mathrm{C}-\mathrm{SWNT}]$. The growth of this chain structure appears to be relatively kinetically favourable and occurs through the same rotation mechanism outlined above for the narrower nanotube. The chain structure then acts as an effective backbone template precursor for the growth of the more space-filling crystallite. The additional free volume available in the $(18,0)$ tube, when compared with the $(16,0)$, allows an additional cation-centred polyhedron to be accommodated in the nanotube, increasingly distorting the initially formed octahedra into trigonal bipyramids. At $\sim 91 \mathrm{ps}$, cation- 
centred polyhedra have entered the tube to form the first section of the final structure. At $\sim 136 \mathrm{ps}$, this second row of cation-centred polyhedra has extended further into the nanotube. At $\sim 145 \mathrm{ps}$, an intermediate structure, also based on two cations, is formed; this structure is related to the final observed filling fragment inside the $(18,0) \mathrm{C}$-SWNT, displayed in Fig. 2(b) (see above). While in both structures the cations are staggered only slightly out of the aligned ladder arrangement, the latter exhibits a regular tendency towards staggering, while the intermediate structure shows random staggering of the cations with respect to each other. Both fragments consist of trigonal biprisms, although they are partially capped and more heavily distorted in the case of the intermediate fragment.

\subsection{Factors controlling formation}

In order to understand the thermodynamic factors controlling the formation of the crystallites, a series of energy minimizations, as a function of carbon nanotube diameter, are performed starting from the ideal crystal structures. Three different crystallites are considered which are derived from the structures observed from the dynamic filling simulations. In addition to the single- and double-chained structures formed in the small $(16,0)$ and medium $(18,0)$ carbon nanotube diameters, the 'ladder' structure is also considered in order to understand how it is related to the 'staggered' configuration. The crystallites constructed from ideal unit cells are extracted from the dynamic structures. These crystallites are then confined in a carbon nanotube of the required radius and the total energy is minimized using a steepest descent algorithm.

Fig. 6 shows the energies of the isolated single-cation chain and the double chain ('staggered' and 'ladder' fragments) as a function of the carbon nanotube diameter. The total energies are shown decomposed into the ion-ion energy, $U^{i i}$, and the ion-carbon energy, $U^{L J}$. At the $(16,0)$ C-SWNT diameter (i.e. $12.53 \AA$ ), the single-chain structure is predicted to be stable over the double-chain structure, whilst the converse is true at the $(18,0)$ diameter (i.e. $14.09 \AA)$. This behaviour can be rationalized in terms of the compressive effect of the nanotube. At small radii, the double-chain structure is heavily compressed compared with the (narrower) single-chain structure and hence becomes thermodynamically unstable. The difference in energetics between the staggered and ladder configurations is more subtle. The total energies indicate that the ladder structure is favoured at carbon nanotube diameters below $\sim 14 \AA$. In order to further understand this behaviour, we consider the breakdown of the total energy into $U^{i i}$ and $U^{L J}$. For $U^{i i}$, the ladder structure is favoured over the staggered over the range of carbon nanotube diameters considered and as a free crystal (as indicated by the $D \longrightarrow \infty$ limit). This trend is explicable as, in the staggered conformation, the cation is able to adopt a bulk-crystal-like local anion coordination environment whilst, in the ladder configuration, the cation is effectively under-coordinated. For $U^{L J}$, however, the interaction energy of the ladder structure with the carbon nanotube appears more favourable compared with the staggered configuration reflecting the smaller effective diameter of the ladder structure. Overall, therefore, although the unconfined ladder structure is unfavourable over the staggered, the more favourable Lennard-Jones energy renders the ladder configuration thermodynamically favourable at smaller nanotube diameters.

The inset to Fig. 6 shows the $0 \mathrm{~K}$ energy/volume curves calculated for four $M X_{3}$ crystal structures $\left(\mathrm{UCl}_{3}, \mathrm{PuBr}_{3}, \mathrm{YCl}_{3}\right.$ and $\mathrm{ZrI}_{3}$ ). As would be predicted experimentally for $\mathrm{LaCl}_{3}$, the $\mathrm{UCl}_{3}$ structure appears thermodynamically stable. However, the $\mathrm{PuBr}_{3}$ structure is found to be very close in energy (with a difference in energy at the respective minima of $\sim 25 \mathrm{~kJ} \mathrm{~mol}^{-1}$ ). The single- and double-chain cation crystallites have clear bulk-crystal analogues which may be obtained by extracting sections of those bulk crystals (and whose bulk energies lie within $\sim 200 \mathrm{~kJ} \mathrm{~mol}^{-1}$ as shown in Fig. 6). For the three-cation chain structure, however, this analysis breaks down and, although the obtained structure can be related to a known bulk crystal (the $\mathrm{UCl}_{3}$ structure), it cannot be produced simply by cutting a section of the bulk crystal. Overall, therefore, the known bulk structures indicate which local ion geometries may be favourable but do not provide definite templates for the observed low-dimensional structures.

We return finally to the step-like behaviour in Fig. 1 (which also shows the relative lack of space filling of the favoured crystallites). The requirement for the crystallites filling the nanotube to be charge neutral effectively 'quantizes' the structures that are reasonably energetically accessible. Fig. 6, therefore, effectively represents the 'energy landscape' available as a function of carbon nanotube diameter.

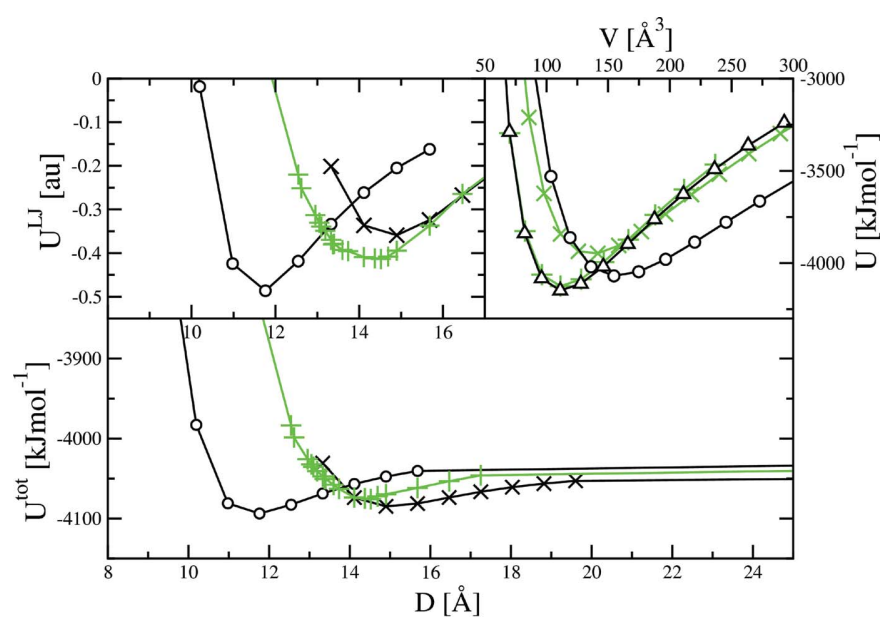

Figure 6

Bottom panel: Total crystallite energies, as a function of enclosing carbon nanotube diameter, for the single-cation centred chain $(\circ)$ and the 'staggered' $(x)$ and 'ladder' $(+)$ double-chain structures. Top left panel: The Lennard-Jones C-ion interaction energies as a function of nanotube diameter. The key is as for the main panel. Top right panel: Energy/ volume curves for four crystal structures of $M X_{3}$ stoichiometry. Key: $\triangle \mathrm{UCl}_{3}$, ○ $\mathrm{YCl}_{3},+\mathrm{PuBr}_{3}, \times \mathrm{ZrI}_{3}$. 


\section{Conclusions}

In conclusion, molecular dynamics simulation models, which utilize existing (relatively simple) potential models for a typical metal trichloride $\left(\mathrm{LaCl}_{3}\right)$ have been shown to reproduce the morphologies of crystallites experimentally observed to form within single-walled carbon nanotubes. Whilst the majority of the crystallites formed have structures that can be understood in terms of known bulk crystal fragments, additional structures have been predicted to exist which do not have such a direct bulk analogue. The use of simulation models of this type allows for the understanding of the basic filling mechanisms, information that is not directly accessible from experimental studies.

The work was supported by EPSRC grant GR/S06233/01. SF is grateful to Professor M. L. H. Green for his exceptional help and encouragement, and to Professor A. I. Kirkland and Dr R. R. Meyer for the development of the digital image restoration codes. Additional thanks go to $\mathrm{C}$. $\mathrm{Xu}$ for the preparation of the LaI $@$ @WNT composite and Dr J. Sloan for the acquisition of the TEM focal series. Financial support from the Cambridge MIT Institute (CMI) is gratefully acknowledged.

\section{References}

Ajayan, P. M. \& Ebbesen, T. W. (1997). Rep. Prog. Phys. 60, 1025-1062.

Ajayan, P. M., Ebbesen, T. W., Ichihashi, T., Iijima, S., Tanigaki, K. \& Hiura, H. (1993). Nature (London), 362, 522-525.

Ajayan, P. M., Ichihashi, T. \& Iijima, S. (1993). Chem. Phys. Lett. 202, 384-388.

Ajayan, P. M., Stephan, O., Redlich, Ph. \& Colliex, C. (1995). Nature (London), 375, 564-567.

Bethune, D. S., Klang, C. H., de Vries, M. S., Gorman, G., Savoy, R., Vasquez, J. \& Beyers, R. (1993). Nature (London), 363, 605-607.

Boothroyd, C. B. (1998). J. Microsc. 190, 99-108.

Chen, Y. K., Green, M. L. H. \& Tsang, S. C. (1996). Chem. Commun. pp. 2489-2490.

Cowley, J. M. \& Moodie, A. F. (1957). Acta Cryst. 10, 609-619.

Friedrichs, S., Falke, U. \& Green, M. L. H. (2005). ChemPhysChem, 6 , 300-305.

Friedrichs. S. \& Green, M. L. H. (2005). Z. Metallkd. 96, 419-426.

Friedrichs, S., Kirkland, A. I., Meyer, R. R., Sloan, J. \& Green, M. L. H. (2004). Electron Microsc. Anal. pp. 455-458.

Friedrichs, S., Meyer, R. R., Sloan, J., Kirkland, A. I., Hutchison, J. L. \& Green, M. L. H. (2001). Chem. Commun. pp. 929-930.

Glover, W. J. \& Madden, P. A. (2004). J. Chem. Phys. 121, 7293-7303.
Goodman, P. \& Moodie, A. F. (1974). Acta Cryst. A30, 280-290.

Hutchinson, F., Rowley, A. J., Walters, M. K., Wilson, M., Madden, P. A., Wasse, J. C. \& Salmon, P. S. (1999). J. Chem. Phys. 111, 2028-2037.

Hutchinson, F., Wilson, M. \& Madden, P. A. (2001). Mol. Phys. 99, 811-824.

lijima, S. (1991). Nature (London), 354, 56-58.

Kirkland, E. (1998). Advanced Computing in Electron Microscopy. New York: Plenum Press.

Larsen, E. M., Wrazel, J. S. \& Hoard, L. G. (1982). Inorg. Chem. 21 2619-2624.

Madden, P. A. \& Wilson, M. (1996). Chem. Soc. Rev. 25, 339-346.

Madden, P. A. \& Wilson, M. (2000). J. Phys. Condens. Matter, 12, A95-A108.

Meyer, R. R., Sloan, J., Dunin-Borkowski, R. E., Kirkland, A. I., Novotny, M. C., Bailey, S. R., Hutchison, J. L. \& Green, M. L. H. (2000). Science, 289, 1324-1326.

Okamoto, Y. \& Madden, P. A. (2005). J. Phys. Chem. Solids, 66, 448-451.

Pettifor, D. G. (1994). Intermetallic Compounds, ch. 18. New York: Wiley.

Philp, E., Sloan, J., Kirkland, A. I., Meyer, R. R., Friedrichs, S., Hutchison, J. L. \& Green, M. L. H. (2003). Nature (Materials), 2, 788-791.

Saito, R., Dresselhaus, G. \& Dresselhaus, M. S. (1998). Physical Properties of Carbon Nanotubes. London: Imperial College Press.

Schleid, T., Meyer, G. \& Morss, L. R. (1987). J. Less-Common Met. 132, 69-77.

Sloan, J., Cook, J., Chu, A., Zwiefka-Sibley, M., Green, M. L. H. \& Hutchison, J. L. (1998). J. Solid State Chem. 140, 83-90.

Sloan, J., Grosvenor, S. J., Friedrichs, S., Kirkland, A. I., Hutchison, J. L. \& Green, M. L. H. (2002). Angew. Chem. Int. Ed. Engl. 41, 1156.

Sloan, J., Novotny, M. C., Bailey, S. R., Brown, G., Xu, C., Williams, V. C., Friedrichs, S., Flahaut, E., Callendar, R. L., York, A. P. E., Coleman, K. S., Green, M. L. H., Dunin-Borkowski, R. E. \& Hutchison, J. L. (2000). Chem. Phys. Lett. 329, 61-65.

Sloan, J., Terrones, M., Nufer, S., Friedrichs, S., Bailey, S. R., Woo, H.-G., Rühle, M., Hutchison, J. L. \& Green, M. L. H. (2002). J. Am. Chem. Soc. 124, 2116-2117.

Sloan, J., Wright, D. M., Woo, H.-G., Bailey, S. R., Brown, G., York, A. P. E., Coleman, K. S., Hutchison, J. L. \& Green, M. L. H. (1999). Chem. Commun. pp. 699-700.

Tsang, S. C., Chen, Y. K., Harris, P. J. F. \& Green, M. L. H. (1994). Nature (London), 372, 159-162.

Wasse, J. C. \& Salmon, P. S. (1999). J. Phys. Condens. Matter, 11, 1381-1396.

Wells, A. F. (1984). Structural Inorganic Chemistry. Oxford: Clarendon Press.

Wilson, M. (2002a). J. Chem. Phys. 116, 3027-3041.

Wilson, M. (2002b). Chem. Phys. Lett. 366, 504-509.

Wilson, M. (2004). Nano. Lett. 4, 299-302.

Wilson, M. \& Madden, P. A. (2001). J. Am. Chem. Soc. 123, 2101-2102.

Zachariasen, W. H. (1948). Acta Cryst. 1, 265-269. 